Corrigendum: Inhibition of JAK-STAT signaling stimulates adult satellite cell function

Feodor D Price, Julia von Maltzahn, C Florian Bentzinger, Nicolas A Dumont, Hang Yin, Natasha C Chang, David H Wilson, Jérôme Frenette \& Michael A Rudnicki

Nat. Med.; doi:10.1038/nm.3655; corrected online 22 September 2014

In the version of this article initially published online, the third sentence of the Abstract read "Gene expression analysis identified higher expression of JAK-STAT signaling targets in 3-week-old relative to 18-month-old mice," when it should have read "Gene expression analysis identified higher expression of JAK-STAT signaling targets in 18-month-old relative to 3-week-old mice." The error has been corrected for the print, PDF and HTML versions of this article.

\title{
Corrigendum: Translation from a DMD exon 5 IRES results in a functional dystrophin isoform that attenuates dystrophinopathy in humans and mice
}

Nicolas Wein, Adeline Vulin, Maria S Falzarano, Christina Al-Khalili Szigyarto, Baijayanta Maiti, Andrew Findlay, Kristin N Heller, Mathias Uhlén, Baskar Bakthavachalu, Sonia Messina, Giuseppe Vita, Chiara Passarelli, Francesca Gualandi, Steve D Wilton, Louise R Rodino-Klapac, Lin Yang, Diane M Dunn, Daniel R Schoenberg, Robert B Weiss, Michael T Howard, Alessandra Ferlini \& Kevin M Flanigan

Nat. Med.; doi:10.1038/nm.3628; corrected 25 August 2014

In the version of this article initially published online, the nucleotide numbering of the AS $(-3$ to +16$), \mathrm{B}(+17$ to +40$)$ and C (+49 to -10$)$ targeted sequences in Figure $3 \mathrm{~b}$ was incorrect, and thus the schematic of the sequences was incorrectly drawn. The correct numbering is as follows: AS, -3 to $+18 ; \mathrm{B},+17$ to +44 ; and $\mathrm{C},+49$ to -8 . The correction has no impact on the results of the study or its conclusions. The error has been corrected for all versions of this article.

Corrigendum: Asfotase- $\alpha$ improves bone growth, mineralization and strength in mouse models of neurofibromatosis type-1

Jean de la Croix Ndong, Alexander J Makowski, Sasidhar Uppuganti, Guillaume Vignaux, Koichiro Ono, Daniel S Perrien, Simon Joubert, Serena R Baglio, Donatella Granchi, David A Stevenson, Jonathan J Rios, Jeffry S Nyman \& Florent Elefteriou Nat. Med. 20, 904-910 (2014); doi:10.1038/nm.3583; published online 6 July 2014; corrected after print 13 March 2015

In the version of this article initially published, the acknowledgment that Daniel S. Perrien was supported by a Career Development Award from the US Department of Veterans Affairs was omitted. The error has been corrected in the HTML and PDF versions of the article.

\section{Erratum: De novo fatty acid synthesis controls the fate between regulatory $T$ and $T$ helper 17 cells}

Luciana Berod, Christin Friedrich, Amrita Nandan, Jenny Freitag, Stefanie Hagemann, Kirsten Harmrolfs, Aline Sandouk, Christina Hesse, Carla N Castro, Heike Bähre, Sarah K Tschirner, Nataliya Gorinski, Melanie Gohmert, Christian T Mayer, Jochen Huehn, Evgeni Ponimaskin, Wolf-Rainer Abraham, Rolf Müller, Matthias Lochner \& Tim Sparwasser

Nat. Med.; doi:10.1038/nm.3704; corrected online 31 October 2014

In the version of this article initially published online, the number 21.45, corresponding to the percentage of cells, was missing from the bottom right quadrant of the flow cytometry plot (SorA, WT) in Figure 2a. The error has been corrected for the print, PDF and HTML versions of this article.

\section{Erratum: A next-generation dual-recombinase system for time- and host- specific targeting of pancreatic cancer}

Nat. Med.; doi:10.1038/nm.3646; corrected online 31 October 2014

In the version of this article initially published online, affiliation 12 was missing for Roland Rad, Roland M. Schmid and Dieter Saur. The list should have read: "Roland Rad ${ }^{1,5,12,13}$, Roland M Schmid ${ }^{1,12,13}$ \& Dieter Saur ${ }^{1,12,13}$ ". The error has been corrected for the print, PDF and HTML versions of this article. 